\title{
La Carta de Jerusalén: un nuevo paradigma en el cuidado de los niños y adolescentes alérgicos en las escuelas de Latinoamérica
}

\author{
Dirceu Solé, ${ }^{1}$ Marilyn Urrutia-Pereira, ${ }^{2}$ Juan Carlos Sisul-Alvariza, ${ }^{3}$ Edgardo J. Jares, ${ }^{4}$ \\ Mario Sánchez-Borges, ${ }^{5}$ Ignacio J. Ansotegui, ${ }^{6}$ Álvaro A. Cruz, ${ }^{7}$ Herberto José Chong-Neto, ${ }^{8}$ \\ Nelson Rosario, ${ }^{8}$ Sandra Nora González-Díaz, ${ }^{9}$ Héctor Badellino, ${ }^{10}$ Marylin Valentín-Rostán, ${ }^{11}$ \\ René Maximiliano Gómez, ${ }^{12}$ Álvaro Teijeiro, ${ }^{13}$ Luis Felipe Ensina, ${ }^{1}$ Renata Cocco, ${ }^{1}$ Roseli Saccardo-Sarni ${ }^{1}$
}

\section{Introducción}

Las enfermedades alérgicas son un problema de salud pública debido a su alta prevalencia e impacto en la calidad de vida, así como por generar importantes gastos económicos y sociales para el paciente, sus familiares y las instituciones de salud. Los procesos alérgicos son variados y pueden presentarse como asma, rinitis alérgica, conjuntivitis alérgica, urticaria, angioedema, dermatitis atópica, alergia a los alimentos, picaduras de insectos y anafilaxia. ${ }^{1}$
De acuerdo con la Organización Mundial de la Salud, las enfermedades alérgicas ocupan el sexto lugar entre las enfermedades infantiles y representan un tercio de las enfermedades crónicas en los niños. En 1 de cada 5 niños, la enfermedad alérgica se manifiesta en la edad escolar. ${ }^{2}$

Por el largo tiempo que los niños pasan dentro de la escuela, es muy importante que el entorno al que están expuestos no sea nocivo. ${ }^{3}$ Las evidencias indican que los profesionales que laboran en las es-

Este artículo debe citarse como: Solé D, Urrutia-Pereira M, Sisul-Alvariza JC, Jares EJ, Sánchez-Borges M, Ansotegui IJ, Cruz ÁA, Chong-Neto HJ, Rosario N, Gonzalez-Díaz SN, Badellino H, Valentín-Rostán M, Gómez-R M, Teijeiro A, Ensina LF, Cocco R, Saccardo-Sarni R. La Carta de Jerusalén: un nuevo paradigma en el cuidado de los niños y adolescentes alérgicos en las escuelas de Latinoamérica. Rev Alerg Mex. 2017;64(1):3-6.

${ }^{1}$ Universidad Federal de São Paulo. São Paulo, Brasil 2Universidad Federal do Pampa. Rio Grande do Sul, Brasil ${ }^{3}$ Hospital Nacional de Itauguá, Departamento de Alergia e Inmunología Clínica. Asunción, Paraguay

${ }^{4}$ Fundación Libra. Buenos Aires, Argentina

${ }^{5}$ Centro Médico Docente La Trinidad, Departamento de Alergia e Inmunología Clínica. Caracas, Venezuela

${ }^{6}$ Hospital Quirónsalud Bizkaia, Departamento de Alergia e Inmunología Clínica. Bilbao, España

${ }^{7}$ Universidade Federal da Bahia. Salvador, Brasil

¿Universidade Federal do Paraná. Curitiba, Brasil

${ }^{9}$ Universidad Autónoma de Nuevo León, Hospital Universitario José Eleuterio González, Centro Regional de Alergia e Inmunología Clínica. Monterrey, Nuevo León, México

${ }^{10}$ Universidad de Economía y Ciencias Sociales. San Francisco, Córdoba, Argentina

\author{
${ }^{11}$ Hospital Pereira Rossell, División de Alergia Pediátrica y Medici- \\ na Respiratoria. Montevideo, Uruguay \\ ${ }^{12}$ Fundación Ayre. Salta, Argentina \\ ${ }^{13}$ Hospital Pediátrico del Niño Jesús, División Respiratoria. Córdo- \\ ba, Argentina. \\ Correspondencia: Marilyn Urrutia-Pereira. \\ urrutiamarilyn@gmail.com \\ Recibido: 2016-12-15 \\ Aceptado: 2016-12-17
}


cuelas tienen un conocimiento insuficiente sobre el impacto negativo de las enfermedades alérgicas en la vida de los niños y sus familias. ${ }^{4}$

Los maestros - considerados, entre otras cosas, como agentes promotores de la salud- deben estar entrenados para identificar y atender en forma rápida y eficiente los cuadros alérgicos, habida cuenta que las reacciones alérgicas pueden poner en peligro la vida. La responsabilidad en la difusión y aplicación de estos conocimientos debería ser compartida con los equipos locales de salud. ${ }^{5}$ Por lo tanto, el conocimiento de las enfermedades alérgicas, sus síntomas o manifestaciones y reacciones graves deberían ser parte de la currícula de su formación profesional. ${ }^{6}$

Para la correcta orientación de los niños portadores de enfermedades alérgicas en las escuelas es necesaria una acción integrada y coordinada entre los profesionales de la salud, el personal de la escuela, la familia y los organismos gubernamentales..$^{7-11}$

\section{Objetivos de la Carta de Jerusalén}

Los objetivos de la Carta de Jerusalén son:

- Presentar evidencias sobre la necesidad de contar con guías latinoamericanas estandarizadas que aborden el cuidado de los niños con enfermedades alérgicas dentro de la escuela.

- Desarrollar estrategias para concientizar a pacientes, familiares, maestros, profesionales de la salud y la comunidad sobre los beneficios de la implementación de estas guías.

- Alentar a los líderes de opinión latinoamericanos a la aplicación de estas guías en sus respectivos países.

- Desarrollar políticas locales que puedan garantizar el cuidado adecuado de los niños portadores de enfermedades alérgicas en las escuelas.

\section{Barreras}

- Ausencia de guías latinoamericanas de recomendaciones para el cuidado estandarizado de los niños portadores de enfermedades alérgicas en las escuelas.

- Falta de una legislación específica que reglamente la atención adecuada de los niños portadores de estas afecciones en las escuelas.

- Ausencia de una acción conjunta y complementaria entre las familias, maestros, administradores de salud, educación y autoridades que par- ticipan en la protección de los derechos de los niños y adolescentes.

\section{Impacto de la implementación de las guías en las escuelas}

La implementación de las guías tendrá como finalidades:

- Disminuir la frecuencia de exacerbaciones agudas en los niños y adolescentes con enfermedades alérgicas en las escuelas.

- Disminuir el impacto económico de las enfermedades alérgicas en los escolares, sus familias y la sociedad.

- Reducir la frecuencia de las enfermedades alérgicas no diagnosticadas o mal controladas.

- Mejorar la educación sobre el efecto de las enfermedades alérgicas en los niños y adolescentes portadores.

- Facilitar la comunicación entre los agentes de salud y educación para reducir la disparidad de criterios para la atención de las enfermedades alérgicas.

- Mejorar la calidad de vida de los niños y adolescentes portadores de enfermedades alérgicas y sus familiares.

\section{Estrategias}

- Fomentar la inclusión de la promoción de la salud alergológica de niños y adolescentes en el proyecto político pedagógico de la escuela,

- Fomentar la creación de una red de educación que garantice a los maestros y a los funcionarios de las escuelas un mejor conocimiento y capacitación para identificar a los niños y adolescentes portadores en situación de riesgo,

- Poner en práctica las recomendaciones mediante la colaboración entre los funcionarios de la salud, las familias y la escuela.

- Alertar y orientar al personal de las escuelas, a los estudiantes y sus familias acerca de las posibilidades de provocaciones, acoso escolar y ciberacoso que pueden afectar a niños y adolescentes por ser portadores de enfermedades alérgicas. ${ }^{12-15}$

\section{Estrategias de difusión de la Carta de Jerusalén y documentos relacionados}

Deben ser acordes según la población a la que se busque llegar: 
- Profesionales de salud: Divulgación en congresos, conferencias, simposios, cursos específicos, campañas publicitarias en medios de comunicación.

- Directores, maestros y funcionarios de las escuelas: El director de la escuela debe gestionar las aprobaciones necesarias ante las autoridades educativas, para autorizar la intervención de personal docente entrenado.

Los maestros deben estar calificados para identificar y atender rápidamente los cuadros alérgicos, en especial aquellos en los que exista riesgo de vida. Esto solo se puede lograr si están capacitados.

- Pacientes, familiares y comunidad: Es crucial que los mensajes en los medios de comunicación (radio, televisión, periódicos y revistas) sean sencillos y claros para ilustrar al público sobre la importancia del conocimiento y manejo de las enfermedades alérgicas.
- Legisladores: Desarrollar políticas locales para asegurar a las autoridades escolares, la implementación de las guías para el cuidado de los niños y adolescentes portadores de enfermedades alérgicas dentro de las escuelas.

\section{Conclusiones}

Los alergólogos desempeñamos un papel importante en la difusión en las escuelas del conocimiento de las enfermedades alérgicas entre los educadores, los alumnos y sus familias, por lo tanto, es fundamental implementar cambios en las políticas públicas educativas. La forma de hacerlo es concientizando a los docentes, legisladores y la comunidad acerca de la importancia del problema, para que los niños y adolescentes portadores de estas enfermedades sean protegidos en el entorno escolar y se eviten situaciones que puedan poner en peligro su vida.

\section{Referencias}

1. Urrutia-Pereira M, Solé D. El niño alérgico en la escuela. ¿Qué es lo que se necesita saber? Rev Alerg Mex. 2016;63(3):283-392. Disponible en: http://revistaalergia.mx/ojs/index.php/ram/article/view/168

2. Pawankar R, Canonica GW, Holgate ST, Lockey RF, editores. WAO White Book on Allergy. USA: World Allergy Organization; 2011. Disponible en: http://www.worldallergy.org/UserFiles/file/WAO-White-Bookon-Allergy_web.pdf

3. Oeder S, Jörres RA, Weichenmeier I, Pusch G, Schober W, Pfab F, et al. Airborne indoor particles from schools are more toxic than outdoor particles. Am J Respir Cell Mol Biol. 2012;47(5):575-582. DOI: http://dx.doi.org/10.1165/rcmb.2012-01390C

4. Jaramillo Y, Reznik M. Do United States' teachers know and adhere to the national guidelines on asthma management in the classroom? A systematic review. Sci World J. 2015 [internet]: 8 pages. DOI: http:// dx.doi.org/10.1155/2015/624828

5. Polloni L, Lazzarotto F, Toniolo A, Ducolin G, Muraro A. What do school personnel know, think and feel about food allergies? Clin Translat Allergy. 2013;3:39. DOI: http://dx.doi.org/10.1186/2045-7022-3-39

6. Getch $Y Q$, Neuharth-Pritchett $S$. Teacher characteristics and knowledge of asthma. Pub Health Nurs. 2009;26(2):124-133. DOI: http://dx.doi.org/10.1111/j.1525-1446.2009.00763.x

7. Muraro A, Clark A, Beyer K, Borrego LM, Borres M, Lodrup Carlsen KC, et al. The management of the allergic child at school: EAACI/ GA2LEN Task Force on the allergic child at school. Allergy. 2010;65(6):681-689. DOI: http://dx.doi.org/10.1111/j.1398-9995.2010.02343.x

8. Urrutia-Pereira M, To T, Cruz ÁA, Solé D. The school as a health promoter for children with asthma: The purpose of an education programme. Allergol Immunopathol (Madr). 2016 Jul 27. pii: S03010546(16)30064-7. DOI: http://dx.doi.org/10.1016/j.aller.2016.04.002

9. Wagner JO. Rosario N, Potter P, Wahn U, Baena-Cagnani CE. A children's asthma charter. Pediatr Allergy Immunol. 2010:21:1-2. DOI: http://dx.doi.org/10.1111/j.1399-3038.2010.00998.x

10. Coelho AC, de Souza-Machado C, Souza-Machado A. School intervention in asthma for adolescents: it is time to act. Paediatr Respir Rev. 2016 Aug 6. pii:S1526-0542(16)30065-3. DOI: http://dx.doi. org/10.1016/j.prrv.2016.08.001 
11. Carvalho Coelho AC, Barretto Cardoso LS, De Souza-Machado C, Souza-Machado A. The impacts of educational asthma interventions in schools: A systematic review of the literature. Can Respir J. 2016 [internet]: 14 pages. DOI: http://dx.doi.org/10.1155/2016/8476206

12. Polloni L, Gini G, Fiore G, Lazzarotto F, Bonaguro R, Toniolo A, et al. Bullying risk in students with food allergy: schoolteachers' awareness. Pediatr Allergy Immunol. 2016;27(2):225-226. DOI: http://dx.doi. org/10.1111/pai.12486

13. Muraro A, Polloni L, Lazzarotto F, Toniolo A, Baldi I, Bonaguro R, et al. Comparison of bullying of foodallergic versus healthy schoolchildren in Italy. J Allergy Clin Immunol. 2014;134(3):749-751. DOI: http:// dx.doi.org/10.1016/j.jaci.2014.05.043.

14. Lieberman JA, Weiss C, Furlong TJ, Sicherer M, Sicherer SH. Bullying among pediatric patients with food allergy. Ann Allergy Asthma Immunol. 2010;105(4):282-286. DOI: http://dx.doi.org/10.1016/j. anai.2010.07.011

15. Gibson-Young L, Martinasek MP, Clutter M, Forrest J. Are students with asthma at increased risk for being a victim of bullying in school or cyberspace? Findings from the 2011 Florida youth risk behavior survey. J Sch Health. 2014;84(7):429-434. DOI: http://dx.doi.org/10.1111/josh.12167 